\title{
An Inverse Problem on Vakonomic Mechanics
}

\author{
Waldyr M. Oliva \\ Instituto Superior Técnico \\ ISR and Departamento de Matemática \\ Centro de Análise Matemática, Geometria e Sistemas Dinâmicos \\ Av. Rovisco Pais, 1049-001 Lisbon, Portugal \\ email: wamoliva@math.ist.utl.pt
}

\section{Gláucio Terra}

Universidade de São Paulo

Instituto de Matemática e Estatística

Departamento de Matemática

Rua do Matão, 1010 05508-090 São Paulo, Brazil

email: glaucio.terra@gmail.com, glaucio@ime.usp.br

\begin{abstract}
We study a version of the inverse problem of Calculus of Variations in the context of Vakonomic Mechanics.
\end{abstract}

\section{Introduction}

The classical inverse problem of the Calculus of Variations consists in finding conditions under which a given system of differential equations derives from a variational principle. The origins of this problem date back to Helmholtz ([6]), in the end of the 19th century, who sought new applications of the powerful Hamilton-Jacobi method to integrate the equations of Mechanics. Two major contributions to solve this problem were made in the last century: the first, by Douglas in the 1930s in his classic papers ([3], 4]); the second, in the 1980s, by Vinogradov ([19], [20]), Tulczyjew ([17]), Anderson ([1]), Tsujishita ([16]), among others (see references in

Received by the editors January 2009.

The first author wishes to thank Fundação para a Ciência e Tecnologia (Portugal) for the support through Program POCI 2010/FEDER.

The second author wishes to thank Centro de Análise, Geometria e Sistemas Dinâmicos at Instituto Superior Técnico, Lisbon, Portugal, where he was kindly received during the year 2008 and also Coordenação de Aperfeiçoamento de Pessoal de Nivel Superior (Brazil) for the support through process 3952-07-0. 
[1]), who geometrized the problem through the introduction of the so-called variational bicomplex. That is a double complex of differential forms in a Fréchet manifold of infinite jets of sections of a fibered manifold, one of the coboundary operators of which is the classical Euler-Lagrange operator of the Calculus of Variations.

In this paper we study a variation of this problem related to Vakonomic Mechanics. That is a formulation of the equations of Mechanics, based on a variational principle, for systems with constraints in the velocities, introduced by Kozlov in the 1980s (see [7, [8], 9]). The study of this inverse problem was partially motivated by the recent development of HamiltonJacobi methods for Vakonomic Mechanics, as in [5].

The organization of the paper is as follows. In section 2, we state notations and definitions. In section 3, we briefly recall the formulation of Vakonomic Mechanics. We show that the Vakonomic equations for a mechanical system endowed with reonomic (i.e. time-dependent) linear constraints in the velocities and with a non-autonomous Lagrangian may be obtained as the Euler-Lagrange equations of a modified Lagrangian on the first jet bundle of the annihilator of the constraint manifold; this is a key observation that allows one to use the variational bicomplex of this bundle in order to study the inverse problem. Moreover, we show that, under certain regularity conditions on the Lagrangian, the solutions of the Vakonomic equations are integral curves of the Reeb vector field associated to a cosymplectic structure; in particular, for autonomous Lagrangians, we reobtain the well known fact that the Vakonomic equations are Hamiltonian. In section 4 we study the inverse problem which is the main subject of this paper. Let $\mathrm{M}$ be a smooth finite dimensional manifold. Given a system of mixed firstand second-order differential equations, we study conditions under which these equations are the Vakonomic equations induced by a non-autonomous Lagrangian $\mathrm{L}$ defined on $\mathbb{R} \times \mathrm{TM}$.

\section{Notations and Definitions}

In this section we fix some notation for jet bundles and we define the variational bicomplex on the bundle of infinite jets of sections of a fibration. We particularize the definitions for a fibration $\pi: E \rightarrow \mathbb{R}$ over $\mathbb{R}$, where $E$ is a smooth $n$-dimensional manifold. The reader is referred to [12] and [1] for more details on the bundle of infinite jets and the variational bicomplex.

For all $k \in \mathbb{N}, \pi_{k}: \mathrm{J}^{k} \pi \rightarrow \mathbb{R}$ denotes the bundle of $k$-jets of sections of $\pi$ and, for $0 \leqslant l<k, \pi_{k, l}: \mathrm{J}^{k} \pi \rightarrow \mathrm{J}^{l} \pi$ denotes the natural projections (where $\left.\mathrm{J}^{0} \pi \doteq \mathrm{E}\right)$. We call $\pi_{1}$ the source projection and $\pi_{1,0}$ the target projection of the 1 st jet bundle $\mathrm{J}^{1} \pi$. Let $\left(t, u^{\alpha}\right)_{1 \leqslant \alpha \leqslant n}$ be coordinates on an open set $\mathcal{U} \subset \mathrm{E}$ adapted to the fibration $\mathrm{E} \rightarrow \mathbb{R}$. This coordinate system induce

São Paulo J.Math.Sci. 5, 1 (2011), 23 35 
coordinates $\left(t, u_{(i)}^{\alpha}\right)_{1 \leqslant \alpha \leqslant n, 0 \leqslant i \leqslant k}$ on $\mathcal{U}^{k} \doteq \pi_{k, 0}^{-1} \mathcal{U} \subset \mathrm{J}^{k} \pi$. We use the notation $\dot{u}^{\alpha} \doteq u_{(1)}^{\alpha}$ and $\ddot{u}^{\alpha} \doteq u_{(2)}^{\alpha}$, so that $\left(t, u^{\alpha}, \dot{u}^{\alpha}\right)_{1 \leqslant \alpha \leqslant n}$ and $\left(t, u^{\alpha}, \dot{u}^{\alpha}, \ddot{u}^{\alpha}\right)_{1 \leqslant \alpha \leqslant n}$ are coordinates on $\mathcal{U}^{1} \subset \mathrm{J}^{1} \pi$ and $\mathcal{U}^{2} \subset \mathrm{J}^{2} \pi$, respectively.

Let $s: I \subset \mathbb{R} \rightarrow \mathrm{E}$ be a smooth section of $\pi$. Given $t \in I$, we denote by $\mathrm{j}_{t}^{\infty} s$ the equivalence class of all sections of $\pi$ defined on a neighborhood of $t$ whose derivatives of all orders at $t$ coincide with that of $s$. Such an equivalence class is called an infinite order jet. We denote by $\mathrm{J}^{\infty} \pi$ the Fréchet manifold of infinite order jets of sections of $\pi$; it is a smooth manifold modelled on the Fréchet space $\mathbb{R}^{\infty}$. We denote by $\left(\forall k \in \mathbb{Z}_{+}\right)$ $\pi_{\infty, k}: \mathrm{J}^{\infty} \pi \rightarrow \mathrm{J}^{k} \pi$ and $\pi_{\infty}: \mathrm{J}^{\infty} \pi \rightarrow \mathbb{R}$ the natural projections. The chart $\left(t, u^{\alpha}\right)_{1 \leqslant \alpha \leqslant n}$ on $\mathcal{U} \subset \mathrm{E}$ adapted to the fibration $\mathrm{E} \rightarrow \mathbb{R}$ induces a chart $\left(t, u_{(i)}^{\alpha}\right)_{1 \leqslant \alpha \leqslant n, 0 \leqslant i<\infty}$ on $\mathcal{U}^{\infty} \doteq \pi_{\infty, 0}^{-1} \mathcal{U} \subset \mathrm{J}^{\infty} \pi$.

We say that a smooth function on $\mathrm{J}^{\infty} \pi$ has order $k \in \mathbb{Z}_{+}$if it is the pullback by $\pi_{\infty, k}$ of a smooth function on $\mathrm{J}^{k} \pi$. We say that a smooth function on $\mathrm{J}^{\infty} \pi$ is of finite order if, for some $k \in \mathbb{Z}_{+}$, it has order $k$. Differential forms on $J^{\infty} \pi$ of finite order are similarly defined. In this paper, all smooth functions or differential forms on $\mathrm{J}^{\infty} \pi$ are assumed to be of finite order.

There exists a natural bigrading on the $\mathbb{R}$-vector space of differential forms on $\mathrm{J}^{\infty} \pi$ :

$$
\Omega_{*}\left(\mathrm{~J}^{\infty} \pi\right)=\underset{0 \leqslant i \leqslant 1,0 \leqslant j<\infty}{\oplus} \Omega_{i, j}\left(\mathrm{~J}^{\infty} \pi\right) .
$$

A differential form belongs to $\Omega_{i, j}\left(\mathrm{~J}^{\infty} \pi\right)$ if, locally, on the charts described above, it is a sum of terms of the form $f \mathrm{~d} t^{i} \wedge \delta u_{\left(k_{1}\right)}^{\alpha_{1}} \wedge \cdots \wedge \delta u_{\left(k_{j}\right)}^{\alpha_{j}}$, where $f$ is a smooth function on $\mathrm{J}^{\infty} \pi$ and $\delta u_{(k)}^{\alpha} \doteq \mathrm{d} u_{(k)}^{\alpha}-u_{(k+1)}^{\alpha} \mathrm{d} t$. Such a form is said to be of type $(i, j)$, or $i$-horizontal and $j$-vertical. Given $\omega \in \Omega_{i, j}\left(\mathrm{~J}^{\infty} \pi\right)$, its exterior derivative $\mathrm{d} \omega$ belongs to $\Omega_{i+1, j}\left(\mathrm{~J}^{\infty} \pi\right) \oplus \Omega_{i, j+1}\left(\mathrm{~J}^{\infty} \pi\right)$; we denote by $\mathrm{d}_{\mathrm{h}} \omega$ its projection on the first factor and by $\mathrm{d}_{\mathrm{v}} \omega$ its projection on the second one. We extend $\mathrm{d}_{\mathrm{h}}$ and $\mathrm{d}_{\mathrm{v}}$ to $\Omega_{*}\left(\mathrm{~J}^{\infty} \pi\right)$ by linearity; $\mathrm{d}_{\mathrm{h}}$ is called horizontal derivative and $\mathrm{d}_{\mathrm{v}}$ is called vertical derivative. They are both anti-derivations on $\Omega_{*}\left(\mathrm{~J}^{\infty} \pi\right)$ of degree +1 and satisfy $\mathrm{d}_{\mathrm{h}}{ }^{2}=0, \mathrm{~d}_{\mathrm{v}}{ }^{2}=0$, $\mathrm{d}_{\mathrm{h}} \mathrm{d}_{\mathrm{v}}=-\mathrm{d}_{\mathrm{v}} \mathrm{d}_{\mathrm{h}}$. Therefore, for each fixed $i$ we obtain a cochain complex $\left(\Omega_{i, j}\left(\mathrm{~J}^{\infty} \pi\right), \mathrm{d}_{\mathrm{v}}\right)_{j \geqslant 0}$ - the columns of the so-called variational bicomplex and for each fixed $j$ we obtain a cochain complex $\left(\Omega_{i, j}\left(\mathrm{~J}^{\infty} \pi\right), \mathrm{d}_{\mathrm{h}}\right)_{i \geqslant 0}$ - the lines of the variational bicomplex. The horizontal and vertical derivatives can be easily computed in coordinates: if $f=f\left(t, u^{\alpha}, u_{(1)}^{\alpha}, \ldots, u_{(k)}^{\alpha}\right)$ is a 
smooth function on $\mathrm{J}^{\infty} \pi$, we have:

$$
\begin{aligned}
& \mathrm{d}_{\mathrm{h}} f=\mathrm{D}_{\mathrm{t}} f \mathrm{~d} t \\
& \mathrm{~d}_{\mathrm{v}} f=\sum_{\alpha=1}^{n} \sum_{j=0}^{k} \frac{\partial f}{\partial u_{(j)}^{\alpha}} \delta u_{(j)}^{\alpha},
\end{aligned}
$$

where $\mathrm{D}_{\mathrm{t}} f=\frac{\partial f}{\partial t}+\sum_{\alpha=1}^{n} \sum_{j=0}^{k} \frac{\partial f}{\partial u_{(j)}^{\alpha}} u_{(j+1)}^{\alpha}$ is the total derivative of $f$; moreover, $\mathrm{d}_{\mathrm{h}} \mathrm{d} t=0=\mathrm{d}_{\mathrm{v}} \mathrm{d} t$ and $\mathrm{d}_{\mathrm{h}} \delta u_{(k)}^{\alpha}=\mathrm{d} t \wedge \delta u_{(k+1)}^{\alpha}, \mathrm{d}_{\mathrm{v}} \delta u_{(k)}^{\alpha}=0$.

The differential forms in $\Omega_{1,0}\left(\mathrm{~J}^{\infty} \pi\right)$ are called Lagrangian forms. In coordinates, a Lagrangian form may be written as $\mathrm{L} d t$, where $\mathrm{L}$ is a smooth function on $\mathrm{J}^{\infty} \pi$; the Lagrangian is said to be of order $k$ if $\mathrm{L}$ is of order $k$, i.e. if $\mathrm{L}=\mathrm{L}\left(t, u^{\alpha}, u_{(1)}^{\alpha}, \ldots, u_{(k)}^{\alpha}\right)$. Such a Lagrangian induces a functional on compactly supported smooth sections of $\pi: s \mapsto \int_{\mathbb{R}}\left(\mathrm{j}^{\infty} s\right)^{*} \mathrm{~L} \mathrm{~d} t$. A smooth section $s$ of $\pi$ is said to be an extremal of the functional induced by $\mathrm{Ld} t$ if, for any compactly supported variation $s_{\tau}$ of $s$, we have $\left.\frac{d}{d \tau}\right|_{\tau=0} \int_{\mathbb{R}}\left(\mathrm{j}^{\infty} s_{\tau}\right)^{*} \mathrm{~L} \mathrm{~d} t=0$. A necessary and sufficient condition for $s$ to be an extremal of the functional induced by $\mathrm{Ld} t$ is that the Euler-Lagrange form $\Omega_{\mathrm{L}}$ of $\mathrm{L}$ be null along $\mathrm{j}^{\infty} \mathrm{s}$. The Euler-Lagrange form $\Omega_{\mathrm{L}}$ is a form in $\Omega_{1,1}\left(\mathrm{~J}^{\infty} \pi\right)$ which, in coordinates, is written as:

$$
\Omega_{\mathrm{L}}=\sum_{\alpha=1}^{n} \mathrm{E}_{\alpha}(\mathrm{L}) \delta u^{\alpha} \wedge \mathrm{d} t,
$$

where $\mathrm{E}_{\alpha}(\mathrm{L})=\sum_{i=0}^{k}\left(-\mathrm{D}_{\mathrm{t}}\right)^{i} \frac{\partial \mathrm{L}}{\partial u_{(i)}^{\alpha}}$ for a Lagrangian of order $k$.

For $s \geqslant 1$, we call the quotient $\mathcal{F}^{s}\left(\mathrm{~J}^{\infty} \pi\right) \doteq \Omega_{1, s}\left(\mathrm{~J}^{\infty} \pi\right) / \mathrm{d}_{\mathrm{h}} \Omega_{0, s}\left(\mathrm{~J}^{\infty} \pi\right)$ space of type $s$ functional forms. It may be identified with the subspace of $\Omega_{1, s}\left(\mathrm{~J}^{\infty} \pi\right)$ which is the image of the interior Euler operator $I: \Omega_{1, s}\left(\mathrm{~J}^{\infty} \pi\right) \rightarrow$ $\Omega_{1, s}\left(\mathrm{~J}^{\infty} \pi\right)$. In coordinates, we have $I(\omega)=\frac{1}{s} \sum_{\alpha=1}^{n} \delta u^{\alpha} \wedge \mathrm{F}_{\alpha}(\omega)$, where $\left.\mathrm{F}_{\alpha}(\omega) \doteq \sum_{i=0}^{k}\left(-\mathrm{D}_{\mathrm{t}}\right)^{i}\left[\delta u_{(i)}^{\alpha}\right\lrcorner \omega\right]$ if $\omega$ has order $k$. For $s=1$, a differential form in $\Omega_{1,1}\left(\mathrm{~J}^{\infty} \pi\right)$ is a type 1 functional form, also called a source form, if, and only if, it is locally of the form $\sum_{\alpha=1}^{n} P_{\alpha} \mathrm{d} t \wedge \delta u^{\alpha}$, where the $P_{\alpha}$ 's are smooth functions on $\mathrm{J}^{\infty} \pi$. Thus, from (1), the Euler-Lagrange form associated to a Lagrangian is a source form, i.e. it belongs to $\mathcal{F}^{1}\left(\mathrm{~J}^{\infty} \pi\right)$. We think of a source form $\omega \in \mathcal{F}^{-1}\left(\mathrm{~J}^{\infty} \pi\right)$ of order $k$ as an intrinsic definition of a system of $n$ differential equations of order $k$; its solutions are the sections $s$ of $\pi$ such that $\omega$ vanishes along $\mathrm{j}^{\infty} s$.

The spaces $\mathcal{F}^{s}$ are part of a cochain complex, the so-called Euler-Lagrange complex of the fibration $\pi: \mathrm{E} \rightarrow \mathbb{R}$ :

São Paulo J.Math.Sci. 5, 1 (2011), 23 35 


$$
0 \longrightarrow \mathbb{R} \longrightarrow \Omega_{0,0} \stackrel{\mathrm{d}_{\mathrm{h}}}{\longrightarrow} \Omega_{1,0} \stackrel{\mathrm{E}}{\longrightarrow} \mathcal{F}^{1} \stackrel{\delta_{\mathrm{v}}}{\longrightarrow} \mathcal{F}^{2} \stackrel{\delta_{\mathrm{v}}}{\longrightarrow} \cdots
$$

where $\mathrm{E}$ is the Euler operator $\mathrm{Ld} t \mapsto \Omega_{\mathrm{L}}$ given by (1) and $\delta_{\mathrm{v}}: \mathcal{F}^{i} \rightarrow \mathcal{F}^{i+1}$ is the vertical derivative induced on functional forms, given by $\delta_{\mathrm{v}} \doteq I \circ \mathrm{d}_{\mathrm{v}}$.

\section{Vakonomic Mechanics}

We denote by $\xi: \mathbb{R} \times \mathrm{M} \rightarrow \mathbb{R}$ the trivial bundle and $\xi_{1}: \mathrm{J}^{1} \xi \rightarrow \mathbb{R}$ its first jet bundle, which is identified with $\mathbb{R} \times \mathrm{TM}$, where $\tau_{\mathrm{M}}: \mathrm{TM} \rightarrow \mathrm{M}$ denotes the tangent bundle of $\mathrm{M}$. We consider a smooth time-dependent Lagrangian $\mathrm{L}: \mathrm{J}^{1} \xi \rightarrow \mathbb{R}$ and a smooth vector sub-bundle $\Pi: \mathscr{D} \rightarrow \mathbb{R} \times \mathrm{M}$ of the target projection $\xi_{1,0}: \mathbb{R} \times \mathrm{TM} \rightarrow \mathbb{R} \times \mathrm{M}$ (which stands for the reonomic linear constraints) and we denote its annihilator in $\mathbb{R} \times \mathrm{T}^{*} \mathrm{M}$ by $\Pi^{*}: \mathscr{D}^{\perp} \rightarrow \mathbb{R} \times \mathrm{M}$. We denote by $\pi: \mathscr{D}^{\perp} \rightarrow \mathbb{R}$ the natural projection. We call $(\mathrm{M}, \mathscr{D}, \mathrm{L})$ a (linearly) constrained mechanical system. We say that a smooth section $\gamma: I \subset \mathbb{R} \rightarrow \mathbb{R} \times \mathrm{M}$ of $\xi$ is compatible with $\mathscr{D}$ or horizontal with respect to $\mathscr{D}$ if its prolongation $\mathrm{j}^{1} \gamma$ lies in $\mathscr{D}$. There are two natural approaches to formulate equations of motion for constrained mechanical systems yielding solutions which are compatible with $\mathscr{D}$ : (1) nonholonomic mechanics (see [11], 10], [14] and references therein), known as "mechanics of the straightest paths", based on d'Alembert-Chetaev's principle; (2) Vakonomic Mechanics(see [2], [11, [10, [15] and references therein), known as "mechanics of the shortest paths", based on the Hamilton's principle of the stationary action. A particular case of the latter is the so-called subRiemannian geometry.

In this section, we briefly recall how the equations of motion in Vakonomic Mechanics are formulated and we prove that these equations may be obtained as the Euler-Lagrange equations of a modified Lagrangian $\mathscr{L}$ defined on $\mathrm{J}^{1} \pi$, the total space of the first jet bundle of $\pi: \mathscr{D}^{\perp} \rightarrow \mathbb{R}$. Moreover, under certain regularity conditions on the Lagrangian L, we show that the solutions of these equations are the trajectories of a Reeb vector field associated to a certain cosymplectic structure (see theorem 3.1, below).

The action functional induced by $\mathrm{L}$ on compactly supported sections $\gamma: I \subset \mathbb{R} \rightarrow \mathbb{R} \times \mathrm{M}$ of $\xi, I$ an open interval, is defined by $\gamma \mapsto \int_{I} \mathrm{~L} \circ \mathrm{j}^{1} \gamma$. We say that a section $\gamma: I \subset \mathbb{R} \rightarrow \mathbb{R} \times \mathrm{M}$ of $\pi$ is a vakonomic trajectory of $(\mathrm{M}, \mathscr{D}, \mathrm{L})$ if it is a critical point of the action functional on compactly supported variations of $\gamma$ compatible with $\mathscr{D}$. By a compactly supported variation of $\gamma$ we mean a smooth map $\Gamma:(-\delta, \delta) \times I \rightarrow \mathbb{R} \times \mathrm{M}$ such that, for all $s \in(-\delta, \delta), \Gamma_{s} \doteq \Gamma(s, \cdot): I \rightarrow \mathbb{R} \times \mathrm{M}$ is a section of $\xi, \Gamma_{0}=\gamma$ and there exists a compact set $K \subset I$ such that for all $s \in(-\delta, \delta)$ and all $t$ outside $K, \gamma_{s}(t)=\gamma(t)$; we say that such a variation is compatible with $\mathscr{D}$ if, for all $s \in(-\delta, \delta), \Gamma_{s}$ is compatible with $\mathscr{D}$. There are two types 
of vakonomic trajectories (see [10, [15]): the normal, which are associated to a certain system of Euler-Lagrange equations, and the abnormal, which correspond to the critical points of the so-called endpoint map. We propose the following characterization of the normal vakonomic trajectories:

Proposition 3.1. Let $\mathscr{L}: \mathrm{J}^{1} \pi \rightarrow \mathbb{R}$ be defined by:

$$
\mathrm{j}_{t}^{1} \Theta \mapsto \mathrm{L}\left(\mathrm{j}_{t}^{1} \gamma\right)+\left\langle\Theta(t), \mathrm{j}_{t}^{1} \gamma\right\rangle
$$

where $\gamma \doteq \Pi^{*} \circ \Theta$ and $\langle\cdot, \cdot\rangle$ is the canonical pairing between $\mathbb{R} \times \mathrm{TM}$ and $\mathbb{R} \times \mathrm{T}^{*} \mathrm{M}$. Then the normal vakonomic trajectories are projections on $\mathbb{R} \times \mathrm{M}$ of the solutions of the Euler-Lagrange equations of $\mathscr{L}$.

Proof. Let $n=\operatorname{dim} \mathrm{M}$ and let $\left(t, q^{i}\right)_{1 \leqslant i \leqslant n}$ be coordinates on an open set $\mathbb{R} \times \mathcal{U}$ in $\mathbb{R} \times \mathbf{M}$. Let $\left(\theta^{\alpha}\right)_{1 \leqslant \alpha \leqslant n-r}$ be a basis of $\left.\mathscr{D}^{\perp}\right|_{\mathbb{R} \times \mathcal{U}}$, where $r=$ rk $\mathscr{D}$. Let $\left(t, q^{i}, \lambda_{\alpha}\right)$ be the induced coordinates on $\left.\mathscr{D}^{\perp}\right|_{\mathbb{R} \times \mathfrak{U}}$. This coordinate system induces, as described in the previous section, coordinates in $\mathrm{J}^{k} \pi$, $1 \leqslant k \leqslant \infty$. Using these coordinates, $\mathscr{L}: \mathrm{J}^{1} \pi \rightarrow \mathbb{R}$ is locally given by $\mathscr{L}\left(t, q^{i}, \lambda_{\alpha}, \dot{q}^{i}, \dot{\lambda}_{\alpha}\right)=\mathrm{L}\left(t, q^{i}, \dot{q}^{i}\right)+\sum_{i, \alpha} \theta_{i}^{\alpha} \lambda_{\alpha} \dot{q}^{i}$, where $\theta_{i}^{\alpha} \doteq\left\langle\theta^{\alpha}, \frac{\partial}{\partial q^{i}}\right\rangle$. A direct computation then shows that the Euler-Lagrange form of the Lagrangian $\mathscr{L} \mathrm{d} t$ is $\Omega_{\mathscr{L}}=\sum_{i} \mathrm{E}_{i}(\mathscr{L}) \delta q^{i} \wedge \mathrm{d} t+\sum_{\alpha} \mathrm{E}_{\alpha}(\mathscr{L}) \delta \lambda_{\alpha} \wedge \mathrm{d} t$, where:

$$
\begin{aligned}
\mathrm{E}_{i}(\mathscr{L}) & =\frac{\partial \mathscr{L}}{\partial q^{i}}-\mathrm{D}_{\mathrm{t}} \frac{\partial \mathscr{L}}{\partial \dot{q}^{i}} \\
\mathrm{E}_{\alpha}(\mathscr{L}) & =\sum_{i} \theta_{i}^{\alpha} \dot{q}^{i}
\end{aligned}
$$

It then follows that the equations $\mathrm{E}_{i}(\mathscr{L})=0$ and $\mathrm{E}_{\alpha}(\mathscr{L})=0$ coincide with the equations for the vakonomic trajectories as defined in [2].

Definition 3.2. We say that $\mathrm{L}$ is $\mathscr{D}$-regular if $\left.\mathbb{F} \mathrm{L}\right|_{\mathscr{D}}: \mathscr{D} \rightarrow \mathscr{D}^{*}$ is a local diffeomorphism (where $\mathbb{F L}$ denotes the fiber derivative of $\mathrm{L}$, i.e. $\forall\left(t, v_{q}\right) \in$ $\left.\mathbb{R} \times \mathrm{TM}, \mathbb{F} \mathrm{L}\left(t, v_{q}\right) \doteq \mathrm{D}\left(\left.\mathrm{L}\right|_{\mathbb{R} \times \mathrm{T}_{q} \mathrm{M}}\right)\left(t, v_{q}\right) \in \mathbb{R} \times \mathrm{T}_{q}^{*} \mathrm{M}\right)$.

Definition 3.3. The mixed bundle is the Whitney sum $\mathscr{D} \oplus_{\mathbb{R} \times \mathrm{M}} \mathscr{D}^{\perp}$. We define $F: \mathrm{J}^{1} \xi \oplus_{\mathbb{R} \times \mathrm{M}} \mathscr{D}^{\perp} \rightarrow \mathbb{R} \times \mathrm{T}^{*} \mathrm{M}$ by $(v, \Theta)_{(t, q)} \mapsto \mathbb{F L}(v)+\Theta$ and $\left.\bar{F} \doteq F\right|_{\mathscr{D} \oplus_{\mathbb{R} \times M} \mathscr{D}}$.

It is an immediate consequence of the above definitions that $\mathrm{L}$ is $\mathscr{D}$ regular if, and only if, $\bar{F}$ is a local diffeomorphism. Let $\theta$ be the canonical contact form on $\mathbb{R} \times \mathrm{T}^{*} \mathrm{M}, \theta_{\mathrm{L}} \doteq \bar{F}^{*} \theta, \omega_{\mathrm{L}} \doteq-\mathrm{d} \theta_{\mathrm{L}}+\mathrm{d} t \wedge \mathrm{dH}$, where $\mathrm{H}$ : $\mathscr{D} \oplus_{\mathbb{R} \times \mathrm{M}} \mathscr{D}^{\perp} \rightarrow \mathbb{R}$ is given by $(v, \Theta)_{(t, q)} \mapsto \mathrm{L}(v)-\mathbb{F} \mathrm{L}(v) \cdot v$. Then, if $\mathrm{L}$ is $\mathscr{D}$-regular, $\left(\omega_{\mathrm{L}}, \mathrm{d} t\right)$ is a cosymplectic structure on $\mathscr{D} \oplus_{\mathbb{R} \times \mathrm{M}} \mathscr{D}^{\perp}$, where $\mathrm{d} t$ is the canonical volume form on $\mathbb{R}$. Moreover, we have the following:

São Paulo J.Math.Sci. 5, 1 (2011), 23 35 
THEOREM 3.1. If $\mathrm{L}$ is $\mathscr{D}$-regular, the normal vakonomic trajectories are in 1-1 correspondence with the integral curves of the Reeb vector field of the cosymplectic structure $\left(\omega_{\mathrm{L}}, d t\right)$.

Remark 3.4. This is a generalization of the well known fact that, in the autonomous case and under the same regularity hypothesis, the normal vakonomic trajectories are integral curves of a Hamiltonian vector field (see [10], [15]).

Proof. (i) We consider coordinates $\left(t, q^{i}, \dot{q}^{i}\right)$ on $\mathrm{J}^{1} \xi \equiv \mathbb{R} \times \mathrm{TM}$ and $\left(t, q^{i}, p_{i}\right)$ on $\mathbb{R} \times \mathrm{T}^{*} \mathrm{M}$ induced by coordinates $\left(t, q^{i}\right)$ on $\mathrm{M}$. We also consider coordinates $\left(t, q^{i}, \lambda_{\alpha}\right)$ on $\mathscr{D}^{\perp}$ as defined in the proof of proposition 3.1, and corresponding induced coordinates $\left(t, q^{i}, \lambda_{\alpha}, q_{(j)}^{i}, \lambda_{\alpha}^{(j)}\right)_{1 \leqslant i \leqslant n, 1 \leqslant \alpha \leqslant n-r, 1 \leqslant j \leqslant k}$ on $\mathrm{J}^{k} \pi$, for $1 \leqslant k<\infty$. These coordinates induce coordinates $\left(t, q^{i}, \dot{q}^{i}, \lambda_{\alpha}\right)$ on $J^{1} \xi \oplus_{\mathbb{R} \times M} \mathscr{D}^{\perp}$; the points in the domain of this chart which belong to $\mathscr{D} \oplus_{\mathbb{R} \times \mathrm{M}} \mathscr{D}^{\perp}$ are those which satisfy $\sum_{i} \theta_{i}^{\alpha} \dot{q}^{i}=0$, where $\theta_{i}^{\alpha} \doteq\left\langle\theta^{\alpha}, \frac{\partial}{\partial q^{i}}\right\rangle$. With respect to these coordinates, we have:

$$
F\left(t, q^{i}, \dot{q}^{i}, \lambda_{\alpha}\right)=\sum_{i}\left(\frac{\partial \mathrm{L}}{\partial \dot{q}^{i}}\left(t, q^{i}, \dot{q}^{i}\right)+\sum_{\alpha} \theta_{i}^{\alpha} \lambda_{\alpha}\right) \frac{\partial}{\partial p_{i}}
$$

i.e. $F^{*} t=t, F^{*} q^{i}=q^{i}$ and $F^{*} p_{i}=\frac{\partial \mathrm{L}}{\partial \dot{q}^{i}}\left(t, q^{i}, \dot{q}^{i}\right)+\sum_{\alpha} \theta_{i}^{\alpha} \lambda_{\alpha}$.

The Euler-Lagrange form is given by

$$
\Omega_{\mathscr{L}}=\sum_{i} \mathrm{E}_{i}(\mathscr{L}) \delta q^{i} \wedge \mathrm{d} t+\sum_{\alpha} \mathrm{E}_{\alpha}(\mathscr{L}) \delta \lambda_{\alpha} \wedge \mathrm{d} t \in \Omega_{1,1}\left(\mathrm{~J}^{\infty} \pi\right)
$$

where, by (4):

$$
\begin{aligned}
\mathrm{E}_{i}(\mathscr{L})= & \frac{\partial \mathrm{L}}{\partial q^{i}}-\mathrm{D}_{\mathrm{t}} \frac{\partial \mathrm{L}}{\partial \dot{q}^{i}}+\sum_{j, \alpha} \frac{\partial \theta_{j}^{\alpha}}{\partial q^{i}} \lambda_{\alpha} \dot{q}^{j}-\mathrm{D}_{\mathrm{t}}\left\{\sum_{\alpha} \theta_{i}^{\alpha} \lambda_{\alpha}\right\}= \\
= & \frac{\partial \mathrm{L}}{\partial q^{i}}-\frac{\partial^{2} \mathrm{~L}}{\partial t \partial \dot{q}^{i}}-\sum_{j} \frac{\partial^{2} \mathrm{~L}}{\partial q^{j} \partial \dot{q}^{i}} \dot{q}^{j}-\sum_{j} \frac{\partial^{2} \mathrm{~L}}{\partial \dot{q}^{j} \partial \dot{q}^{i}} \ddot{q}^{j}+ \\
& +\sum_{j, \alpha} \frac{\partial \theta_{j}^{\alpha}}{\partial q^{i}} \lambda_{\alpha} \dot{q}^{j}-\sum_{\alpha}\left(\frac{\partial \theta_{i}^{\alpha}}{\partial t}+\sum_{j} \frac{\partial \theta_{i}^{\alpha}}{\partial q^{j}} \dot{q}^{j}\right) \lambda_{\alpha}-\sum_{\alpha} \theta_{i}^{\alpha} \dot{\lambda}_{\alpha} \\
\mathrm{E}_{\alpha}(\mathscr{L})= & \sum_{i} \theta_{i}^{\alpha} \dot{q}^{i}
\end{aligned}
$$

A section $t \in I \mapsto \Theta(t)=\left(t, q^{i}(t), \lambda_{\alpha}(t)\right)$ of $\pi$ is a normal vakonomic trajectory if, and only if, the equations $\mathrm{E}_{i}(\mathscr{L})=0$ and $\mathrm{E}_{\alpha}(\mathscr{L})=0$ are satisfied 
along $\mathrm{j}^{\infty} \Theta$. Denoting $\gamma \doteq \Pi^{*} \Theta$, it therefore follows that $\gamma: I \rightarrow \mathbb{R} \times \mathrm{M}$ is compatible with $\mathscr{D}$ and $\frac{d}{d t}\left\{\frac{\partial \mathrm{L}}{\partial \dot{q}^{i}}\left(t, q^{i}(t), \dot{q}^{i}(t)\right)+\sum_{\alpha} \theta_{i}^{\alpha}\left(t, q^{i}(t)\right) \lambda_{\alpha}(t)\right\}=$ $\frac{\partial \mathrm{L}}{\partial q^{i}}\left(t, q^{i}(t), \dot{q}^{i}(t)\right)+\sum_{j, \alpha} \frac{\partial \theta_{j}^{\alpha}}{\partial q^{i}}\left(t, q^{i}(t)\right) \lambda_{\alpha}(t) \dot{q}^{j}(t)$ for $1 \leqslant i \leqslant n$. Thus, defining $\Gamma \doteq\left(\mathrm{j}^{1} \gamma, \Theta\right): I \rightarrow \mathscr{D} \oplus_{\mathbb{R} \times \mathrm{M}} \mathscr{D}^{\perp}$, it follows that $\Gamma$ is an integral curve of the vector field $X \in \mathfrak{X}\left(\mathscr{D} \oplus_{\mathbb{R} \times \mathrm{M}} \mathscr{D}^{\perp}\right)$ which, in coordinates, is given by:

$$
\mathrm{T} \bar{F} \cdot X=\frac{\partial}{\partial t}+\sum_{i} \dot{q}^{i} \frac{\partial}{\partial q^{i}}+\sum_{i}\left(\frac{\partial \mathrm{L}}{\partial q^{i}}+\sum_{j, \alpha} \frac{\partial \theta_{j}^{\alpha}}{\partial q^{i}} \lambda_{\alpha} \dot{q}^{j}\right) \frac{\partial}{\partial p_{i}} \in \mathfrak{X}\left(\mathbb{R} \times \mathrm{T}^{*} \mathrm{M}\right)
$$

That $X$ is globally defined follows from the fact that it is the Reeb vector field of the of the cosymplectic structure $\left(\omega_{\mathrm{L}}, \mathrm{d} t\right)$, as we show in part (ii).

Since Т $\Pi \cdot X=\mathrm{T}^{*} \cdot \mathrm{T} \bar{F} \cdot X=\frac{\partial}{\partial t}+\sum_{i} \dot{q}^{i} \frac{\partial}{\partial q^{i}}$, it follows that $X$ is a second order vector field, in the sense that its integral curves are of the form $\Phi \doteq\left(\mathrm{j}^{1} \phi, \eta\right): I \rightarrow \mathscr{D} \oplus_{\mathbb{R} \times \mathrm{M}} \mathscr{D}^{\perp}$, where $\phi: I \rightarrow \mathbb{R} \times \mathrm{M}$ is compatible with $\mathscr{D}$. Moreover, for any such integral curve, $\eta=\operatorname{pr}_{2} \circ \Phi: I \rightarrow$ $\mathscr{D}^{\perp}$ is a normal vakonomic trajectory. We have thus obtained a bijection $\Phi \mapsto \operatorname{pr}_{2} \circ \Phi$ between the integral curves of $X$ and the normal vakonomic trajectories.

(ii) We assert that $X \in \mathfrak{X}\left(\mathscr{D} \oplus_{\mathbb{R} \times M} \mathscr{D}^{\perp}\right)$ is the Reeb vector field of the cosymplectic structure $\left(\omega_{\mathrm{L}}, d t\right)$, i.e. $\left.X\right\lrcorner \omega_{\mathrm{L}}=0$ and $\left.X\right\lrcorner \mathrm{d} t=1$.

Let $\Omega \doteq-\mathrm{d} F^{*} \theta_{\mathrm{L}}+\mathrm{d} t \wedge \mathrm{dH} \in \Omega_{1}\left(\mathrm{~J}^{1} \xi \oplus_{\mathbb{R} \times \mathrm{M}} \mathscr{D}\right)$, where $\mathrm{H}: \mathrm{J}^{1} \xi \oplus_{\mathbb{R} \times \mathrm{M}} \mathscr{D}^{\perp} \rightarrow$ $\mathbb{R}$ is given by $(v, \Theta)_{(t, q)} \mapsto \mathrm{L}(v)-\mathbb{F} \mathrm{L}(v) \cdot v$. Then $\omega_{\mathrm{L}}$ is the pullback of $\Omega$ by the inclusion. We assert that, on $\left.\mathscr{D} \oplus_{\mathbb{R} \times \mathrm{M}} \mathscr{D}^{\perp}, X\right\lrcorner \Omega$ is null. Since it is immediate from (7) that $X\lrcorner \mathrm{d} t=1$, the proof follows from this assertion. Indeed, a direct computation using (7), (5) and the expressions in coordinates $\theta=\sum_{i} p_{i} \mathrm{~d} q^{i}$ and $\mathrm{H}=\mathrm{L}-\sum_{i} \frac{\partial \mathrm{L}}{\partial \dot{q}^{i}} \dot{q}^{i}$ yields, on $\mathscr{D} \oplus_{\mathbb{R} \times \mathrm{M}} \mathscr{D}^{\perp}$ :

$$
\begin{aligned}
X\lrcorner \Omega= & \sum_{i} \dot{q}^{i} \mathrm{~d}\left(\frac{\partial \mathrm{L}}{\partial \dot{q}^{i}}+\sum_{\alpha} \theta_{i}^{\alpha} \lambda_{\alpha}\right)-\sum_{i}\left(\frac{\partial \mathrm{L}}{\partial q^{i}}+\sum_{j, \alpha} \frac{\partial \theta_{j}^{\alpha}}{\partial q^{i}} \lambda_{\alpha} \dot{q}^{j}\right) \mathrm{d} q^{i}+ \\
& +\mathrm{d}\left(\mathrm{L}-\frac{\partial \mathrm{L}}{\partial \dot{q}^{i}} \dot{q}^{i}\right)-\langle\mathrm{dH}, X\rangle \mathrm{d} t= \\
= & \left(\frac{\partial \mathscr{L}}{\partial t}-\langle\mathrm{dH}, X\rangle\right) \mathrm{d} t+\sum_{i, \alpha} \theta_{i}^{\alpha} \dot{q}^{i} \mathrm{~d} \lambda_{\alpha}
\end{aligned}
$$

São Paulo J.Math.Sci. 5, 1 (2011), 23 35 
Another direct computation in coordinates yields $\frac{\partial \mathscr{L}}{\partial t}-\langle\mathrm{dH}, X\rangle=0$. Since, for all $\alpha, \sum_{i} \theta_{i}^{\alpha} \dot{q}^{i}=0$ on $\mathscr{D} \oplus_{\mathbb{R} \times \mathrm{M}} \mathscr{D}^{\perp}$, the assertion is proved and the thesis follows.

\section{The Inverse Problem}

We consider a source form $\Omega$ on $\mathcal{F}^{1}\left(\mathrm{~J}^{\infty} \pi\right)$ of order 2 which, on the coordinates introduced in the proof of proposition 3.1, is of the form:

$$
\sum_{i} P_{i}\left(t, q^{i}, \dot{q}^{i}, \ddot{q}^{i}, \lambda_{\alpha}, \dot{\lambda}_{\alpha}\right) \delta q^{i} \wedge \mathrm{d} t+\sum_{i, \alpha} \theta_{i}^{\alpha} \dot{q}^{i} \delta \lambda_{\alpha} \wedge \mathrm{d} t
$$

where $\theta_{i}^{\alpha} \doteq\left\langle\theta^{\alpha}, \frac{\partial}{\partial q^{i}}\right\rangle$.

Definition 4.1. With the notation above, we call $\Omega$ a $\mathscr{D}$-source form. The integral curves of $\Omega$ are the sections $\Theta$ of $\pi$ such that $\Omega$ vanishes along $\mathrm{j}^{\infty} \Theta$.

Proposition 4.2. The definition above is intrinsic, i.e. independent of the coordinate system.

Proof. Let $\left(t, \bar{q}^{i}\right)_{1 \leqslant i \leqslant n}$ be another coordinate system on $\mathbb{R} \times \mathcal{U} \subset \mathbb{R} \times \mathrm{M}$; we assume these coordinate systems to be related by $\left(t, q^{i}\right) \mapsto\left(t, \bar{q}^{i}\left(q^{1}, \ldots, q^{n}\right)\right)$. Let $\left(\bar{\theta}^{\alpha}\right)_{1 \leqslant \alpha \leqslant n-k}$ be another basis of $\left.\mathscr{D}^{\perp}\right|_{\mathbb{R} \times u}$; we assume $\bar{\theta}^{\alpha}=\sum_{\beta} A_{\beta}^{\alpha} \theta^{\beta}$, where $A_{\beta}^{\alpha}=A_{\beta}^{\alpha}(t, q)$ is a smooth function on $\mathbb{R} \times \mathcal{U}$ for $1 \leqslant \alpha, \beta \leqslant n-k$. The matrix $A=\left(A_{\beta}^{\alpha}\right)$ is, then, invertible. Let $\left(t, \bar{q}^{i}, \bar{\lambda}_{\alpha}\right)$ be the induced coordinates on $\left.\mathscr{D}^{\perp}\right|_{\mathbb{R} \times u}$. This coordinate system induces coordinates in $\mathrm{J}^{k} \pi$, $1 \leqslant k \leqslant \infty$. A direct computation then shows that, in this new coordinate system, $\Omega$ given by (8) transforms into $\sum_{i} \bar{P}_{i}\left(t, \bar{q}^{i}, \dot{\bar{q}}^{i}, \ddot{\bar{q}}^{i}, \bar{\lambda}_{\alpha}, \dot{\bar{\lambda}}_{\alpha}\right) \delta \bar{q}^{i} \wedge \mathrm{d} t+$ $\sum_{i, \alpha} \bar{\theta}_{i}^{\alpha} \dot{\bar{q}}^{i} \delta \bar{\lambda}_{\alpha} \wedge \mathrm{d} t$, where $\bar{\theta}_{i}^{\alpha} \doteq\left\langle\bar{\theta}^{\alpha}, \frac{\partial}{\partial \bar{q}^{i}}\right\rangle$ and:

$$
\bar{P}_{i}\left(t, \bar{q}^{i}, \dot{\bar{q}}^{i}, \ddot{\bar{q}}^{i}, \bar{\lambda}_{\alpha}, \dot{\bar{\lambda}}_{\alpha}\right)=\sum_{j} P_{j} \frac{\partial q^{j}}{\partial \bar{q}^{i}}+\sum_{j, \alpha, \beta, \gamma} \dot{\bar{q}}^{j} \bar{\theta}_{j}^{\beta}\left(A^{-1}\right)_{\beta}^{\alpha} \frac{\partial A_{\alpha}^{\gamma}}{\partial \bar{q}^{i}} \bar{\lambda}_{\gamma}
$$

Note that, if $\Theta$ is an integral curve of $\Omega$, then the projection of $\Theta$ on $\mathbb{R} \times \mathrm{M}$ is compatible with $\mathscr{D}$ and, locally, $\Theta: t \mapsto\left(t, q^{i}(t), \lambda_{\alpha}(t)\right)$ in the above coordinates is a solution of the system of mixed first- and secondorder equations $P_{i}\left(t, q^{i}, \dot{q}^{i}, \ddot{q}^{i}, \lambda_{\alpha}, \dot{\lambda}_{\alpha}\right)=0$. 
We now consider the following problem: to find necessary and sufficient conditions for a given $\mathscr{D}$-source form $\Omega$ to be the Euler-Lagrange form of a Lagrangian of the form (3). If that is the case, the integral curves of $\Omega$ are the solutions of the Vakonomic equations of the Lagrangian L.

Definition 4.3. We say that a $\mathscr{D}$-source form $\Omega$ is 2-1-affine if, written in coordinates as (8), for $1 \leqslant i \leqslant n$ : (i) the functions $P_{i}$ are affine in the variables $\ddot{q}^{k}, 1 \leqslant k \leqslant n$ and $\dot{\lambda}_{\alpha}, 1 \leqslant \alpha \leqslant n-k$; (ii) $\frac{\partial P_{i}}{\partial \dot{q}^{k}}$ is a function of $(t, q, \dot{q})$ and $\frac{\partial P_{i}}{\partial \dot{\lambda}^{k}}$ is a function of $(t, q)$.

It follows from (9) that the above definition does not depend on the coordinate system.

Definition 4.4. Let $\Omega$ be a $\mathscr{D}$-source form. We say that $\Omega$ is a locally variational $\mathscr{D}$-source form if, locally, it is the Euler-Lagrange form of a Lagrangian of the form (3). We say that $\Omega$ is globally variational if the latter condition holds globally on $\mathrm{J}^{\infty} \pi$.

Our main results are stated in the following theorems: in the first one we describe the $\mathscr{D}$-source forms which are locally variational; in the second one we show that the topological obstruction for a locally variational $\mathscr{D}$-source form to be globally variational lies in $\mathrm{H}^{2}(\mathrm{M})$.

THEOREM 4.1. Let $\Omega$ be a $\mathscr{D}$-source form. Then $\Omega$ is locally variational if, and only if, $\Omega$ is 2-1-affine and $\delta_{\mathrm{v}} \Omega=0$, where $\delta_{\mathrm{v}}$ is defined in (2). In coordinates, if $\Omega$ is given by (8), the latter condition reads:

$$
\begin{aligned}
\frac{\partial P_{j}}{\partial q^{i}} & =\frac{\partial P_{i}}{\partial q^{j}}-\mathrm{D}_{\mathrm{t}} \frac{\partial P_{i}}{\partial \dot{q}^{j}}+\mathrm{D}_{\mathrm{t}}^{2} \frac{\partial P_{i}}{\partial \ddot{q}^{j}} \\
-\frac{\partial P_{j}}{\partial \dot{q}^{i}} & =\frac{\partial P_{i}}{\partial \dot{q}^{j}}-2 \mathrm{D}_{\mathrm{t}} \frac{\partial P_{i}}{\partial \ddot{q}^{j}} \\
\frac{\partial P_{j}}{\partial \ddot{q}^{i}} & =\frac{\partial P_{i}}{\partial \ddot{q}^{j}} \\
\sum_{j} \frac{\partial \theta_{j}^{\alpha}}{\partial q^{i}} \dot{q}^{j} & =\frac{\partial P_{i}}{\partial \lambda_{\alpha}}-\mathrm{D}_{\mathrm{t}} \frac{\partial P_{i}}{\partial \dot{\lambda}_{\alpha}} \\
-\theta_{i}^{\alpha} & =\frac{\partial P_{i}}{\partial \dot{\lambda}_{\alpha}}
\end{aligned}
$$

Proof. The necessity is obvious from (6) and (2). The condition $\delta_{\mathrm{v}} \Omega=0$ in coordinates $(10)$ is obtained by a direct computation using the definition of $\delta_{\mathrm{v}}$ and the canonical form for type 2 functional forms given in [1]; equations (10) are the classical Helmholtz equations for the source form $\Omega$.

São Paulo J.Math.Sci. 5, 1 (2011), 23,35 
Conversely, assume that the $\mathscr{D}$-source form $\Omega$ is 2-1-affine and $\delta_{\mathrm{v}} \Omega=0$. The latter condition implies, by the local exactness of the Euler-Lagrange complex (2) (see 1), the existence of a smooth function $\mathscr{L}$ locally defined on $J^{\infty} \pi$ such that $\Omega$ is, locally, the Euler-Lagrange form of $\mathscr{L}$. We must prove that $\mathscr{L}$ may be taken of the form (3).

Firstly, we may take $\mathscr{L}$ given by the homotopy operator $\mathcal{H}^{1}: \mathcal{F}^{1}\left(\mathrm{~J}^{\infty} \pi\right) \rightarrow$ $\Omega_{1,0}\left(\mathrm{~J}^{\infty} \pi\right)$ defined by the Volterra-Vainberg formula ([21], [18]). Using coordinates defined in section 2 , this homotopy operator is given by:

$$
\sum_{\alpha=1}^{n} f_{\alpha} \delta u^{\alpha} \wedge \mathrm{d} t \mapsto\left(\int_{0}^{1} \sum_{\alpha} u^{\alpha} f_{\alpha}\left(t, s u^{\alpha}, s u_{(1)}^{\alpha}, \ldots, s u_{(k)}^{\alpha}\right) \mathrm{d} s\right) \mathrm{d} t
$$

if the $f_{\alpha}$ 's are smooth functions of order $k$ on $\mathrm{J}^{\infty} \pi$. Thus:

$$
\begin{aligned}
\mathscr{L}(t, q, \dot{q}, \ddot{q}, \lambda, \dot{\lambda})= & \int_{0}^{1} \sum_{i} q^{i} P_{i}\left(t, s q^{i}, s \dot{q}^{i}, s \ddot{q}^{i}, s \lambda_{\alpha}, s \dot{\lambda}_{\alpha}\right) \mathrm{d} s+ \\
& +\int_{0}^{1} \sum_{i, \alpha} \lambda_{\alpha} \theta_{i}^{\alpha}(t, s q) s \dot{q}^{i} \mathrm{~d} s
\end{aligned}
$$

In order to obtain a Lagrangian of the form (3), we now modify the lagrangian form $\Lambda \doteq \mathscr{L} \mathrm{d} t$ (where $\mathscr{L}$ is given by (11)) by the addition of horizontal derivatives of convenient functions on $\mathrm{J}^{\infty} \pi$; since the Euler operator $\mathrm{E}$ is null on the image of $\mathrm{d}_{\mathrm{h}}: \Omega_{0,0}\left(\mathrm{~J}^{\infty} \pi\right) \rightarrow \Omega_{1,0}\left(\mathrm{~J}^{\infty} \pi\right)$, any such modified Lagrangian form has the same Euler-Lagrange form $\Omega$.

Let $f$ be a smooth function on $\mathrm{J}^{\infty} \pi$ which is the pullback of a smooth function on $J^{1} \xi$ (we identify $\mathbb{R} \times M \subset \mathscr{D}^{\perp}$ with the null section of $\Pi^{*}$ : $\mathscr{D}^{\perp} \rightarrow \mathbb{R} \times \mathrm{M}$, so that $\left.\mathrm{J}^{1} \xi \subset \mathrm{J}^{1} \pi\right)$; that is, $f=f(t, q, \dot{q})$. We then have, in coordinates, $\mathrm{d}_{\mathrm{h}} f=\mathrm{D}_{\mathrm{t}} f \mathrm{~d} t=\left(\frac{\partial f}{\partial t}+\sum_{i} \frac{\partial f}{\partial q^{i}} \dot{q}^{i}+\sum_{i} \frac{\partial f}{\partial \dot{q}^{i}} \ddot{q}^{i}\right) \mathrm{d} t$. We define $\Lambda^{\prime}=\mathscr{L}^{\prime} \mathrm{d} t \doteq \Lambda+\mathrm{d}_{\mathrm{h}} f=\left(\mathscr{L}+\mathrm{D}_{\mathrm{t}} f\right) \mathrm{d} t$, so that $\frac{\partial \mathscr{L}^{\prime}}{\partial \ddot{q}^{i}}=\frac{\partial \mathrm{L}}{\partial \ddot{q}^{i}}+\frac{\partial f}{\partial \dot{q}^{i}}$. Since $\Omega$ is 2 -1-affine, $\frac{\partial P_{i}}{\partial \ddot{q}^{k}}$ is a function of $(t, q, \dot{q})$, i.e. it is the pullback of a smooth function on $J^{1} \xi$; hence, differentiating (11) under the integral sign, we conclude that $\frac{\partial \mathscr{L}}{\partial \ddot{q}^{i}}$ is a function of $(t, q, \dot{q})$. Therefore, we may choose $f$ so that $\frac{\partial \mathrm{L}}{\partial \ddot{q}^{i}}+\frac{\partial f}{\partial \dot{q}^{i}}=0$, i.e. $\mathscr{L}^{\prime}=\mathscr{L}^{\prime}(t, q, \dot{q}, \lambda, \dot{\lambda})$.

Let now $f^{\prime}$ be a smooth function on $\mathrm{J}^{\infty} \pi$ which is the pullback of a smooth function on $\mathscr{D}^{\perp}$, that is, $f^{\prime}=f^{\prime}(t, q, \lambda)$, so that $\mathrm{d}_{\mathrm{h}} f^{\prime}=\mathrm{D}_{\mathrm{t}} f^{\prime} \mathrm{d} t=$ $\left(\frac{\partial f^{\prime}}{\partial t}+\sum_{i} \frac{\partial f^{\prime}}{\partial q^{2}} \dot{q}^{i}+\sum_{\alpha} \frac{\partial f^{\prime}}{\partial \lambda_{\alpha}} \dot{\lambda}_{\alpha}\right) \mathrm{d} t$. We define $\Lambda^{\prime \prime}=\mathscr{L}^{\prime \prime} \mathrm{d} t \doteq \Lambda^{\prime}+\mathrm{d}_{\mathrm{h}} f^{\prime}=\left(\mathscr{L}^{\prime}+\right.$ $\left.\mathrm{D}_{\mathrm{t}} f^{\prime \prime}\right) \mathrm{d} t$, so that $\mathscr{L}^{\prime \prime}=\mathscr{L}^{\prime \prime}(t, q, \dot{q}, \lambda, \dot{\lambda})$ and $\frac{\partial \mathscr{L}^{\prime \prime}}{\partial \dot{\lambda}_{\alpha}}=\frac{\partial \mathscr{L}^{\prime}}{\partial \dot{\lambda}_{\alpha}}+\frac{\partial f^{\prime}}{\partial \lambda_{\alpha}}$. Again by the fact that $\Omega$ is 2-1-affine, $\frac{\partial P_{i}}{\partial \dot{\lambda}_{\alpha}}$ is a function of $(t, q)$; hence, differentiating 
once more 11) under the integral sign, we conclude that $\frac{\partial \mathscr{L}^{\prime}}{\partial \dot{\lambda}_{\alpha}}=\frac{\partial \mathscr{L}}{\partial \dot{\lambda}_{\alpha}}$ is a function of $(t, q)$. Then we may choose $f^{\prime}$ so that $\frac{\partial \mathscr{L}^{\prime}}{\partial \dot{\lambda}_{\alpha}}+\frac{\partial f^{\prime}}{\partial \lambda_{\alpha}}=0$, i.e. $\mathscr{L}^{\prime \prime}=\mathscr{L}^{\prime \prime}(t, q, \dot{q}, \lambda)$.

Then $\mathscr{L}^{\prime \prime}$ has Euler-Lagrange form $\Omega$ and $\frac{\partial \mathscr{L}^{\prime \prime}}{\partial \dot{\lambda}_{\alpha}}=0$, so that $\mathrm{E}_{\alpha}\left(\mathscr{L}^{\prime \prime}\right)=$ $\frac{\partial \mathscr{L}^{\prime \prime}}{\partial \lambda_{\alpha}}-\mathrm{D}_{\mathrm{t}} \frac{\partial \mathscr{L}^{\prime \prime}}{\partial \dot{\lambda}_{\alpha}}=\frac{\partial \mathscr{L}^{\prime \prime}}{\partial \lambda_{\alpha}}=\sum_{i, \alpha} \theta_{i}^{\alpha} \dot{q}^{i}$. The last equation implies that, for some smooth function $\mathrm{L}=\mathrm{L}(t, q, \dot{q})$, we have $\mathscr{L}^{\prime \prime}=\mathrm{L}+\sum_{i, \alpha} \theta_{i}^{\alpha} \dot{q}^{i} \lambda_{\alpha}$, i.e. $\mathscr{L}^{\prime \prime}$ is a Lagrangian of the form (3).

THEOREM 4.2. If $\mathrm{H}^{2}(\mathrm{M})=0$, every locally variational $\mathscr{D}$-source form is globally variational. On the other hand, if $\mathrm{H}^{2}(\mathrm{M}) \neq 0$, there exist locally variational $\mathscr{D}$-source forms which are not globally variational.

Proof. Let $\Omega$ on $\mathcal{F}^{1}\left(\mathrm{~J}^{\infty} \pi\right)$ be a locally variational $\mathscr{D}$-source form. Let $\eta \doteq$ $\mathrm{L}^{\prime} \mathrm{d} t \in \Omega_{1,0}\left(\mathrm{~J}^{\infty} \pi\right)$, where $\mathrm{L}^{\prime}$ is the smooth map on $\mathrm{J}^{\infty} \pi$ defined by $\mathrm{j}_{t}^{1} \Theta \mapsto$ $\left\langle\Theta(t), \mathrm{j}_{t}^{1} \gamma\right\rangle$, where $\gamma \doteq \Pi^{*} \circ \Theta$ and $\langle\cdot, \cdot\rangle$ is the canonical pairing between $\mathbb{R} \times \mathrm{TM}$ and $\mathbb{R} \times \mathrm{T}^{*} \mathrm{M}$. As in the previous proof, we identify $\mathbb{R} \times \mathrm{M} \subset \mathscr{D}^{\perp}$ with the null section of $\Pi^{*}: \mathscr{D}^{\perp} \rightarrow \mathbb{R} \times \mathrm{M}$, so that $\mathrm{J}^{k} \xi \subset \mathrm{J}^{k} \pi$ for $0 \leqslant k \leqslant \infty$. By the previous theorem, we may take an open cover $\left(\mathcal{U}_{\alpha}\right)_{\alpha \in A}$ of $\mathrm{J}^{\infty} \pi$ such that, for each $\alpha \in A$, there exists a smooth Lagrangian $\mathrm{L}_{\alpha}$ in $\mathrm{J}^{1} \xi$ such that $\left.\Omega\right|_{\mathcal{U}_{\alpha}}$ is the Euler-Lagrange form of $\mathrm{L}_{\alpha} \mathrm{d} t+\eta$. Hence, on each $\mathcal{U}_{\alpha}$ :

$$
\mathrm{E}\left(\mathrm{L}_{\alpha} \mathrm{d} t\right)=\Omega-\mathrm{E}(\eta)
$$

Since, for each $\mathcal{U}_{\alpha}, \mathrm{L}_{\alpha} \mathrm{d} t$ is a type $(1,0)$ form on an open subset of $\mathrm{J}^{\infty} \xi$, it follows from $(12)$ that the restriction of $\Omega-\mathrm{E}(\eta) \in \mathcal{F}^{1}\left(\mathrm{~J}^{\infty} \pi\right)$ to $\mathrm{J}^{\infty} \xi \subset \mathrm{J}^{\infty} \pi$ is a source form on $\mathrm{J}^{\infty} \xi$ which is locally variational. The total space of the fibration $\xi$ is $\mathbb{R} \times M$, which is contractible to $M$, so that $H^{2}(R \times M) \cong H^{2}(M)$. Therefore, if $H^{2}(M)=0$, we may apply the theory of [13] (see also [1]) to conclude that $\Omega-\mathrm{E}(\eta)$ is a globally variational source form on $\mathrm{J}^{\infty} \xi$. Thus, we may take a globally defined Lagrangian $\mathrm{L}$ in $\mathrm{J}^{1} \xi$ such that $\mathrm{E}(\operatorname{Ld} t)=\Omega-\mathrm{E}(\eta)$, i.e. $\Omega$ is the Euler-Lagrange form of $\operatorname{Ld} t+\eta$, as asserted.

On the other hand, if $\mathrm{H}^{2}(\mathbb{R} \times M) \cong \mathrm{H}^{2}(M) \neq 0$, it follows from the main theorem in [13] that we may take a locally variational source form $\omega$ in $\mathrm{J}^{1} \xi$ which is not globally variational. Therefore, taking the pull back of $\omega$ to $\mathrm{J}^{1} \pi$ by $\Pi^{*}$ (which we also denote by $\omega$ ), we obtain a locally variational $\mathscr{D}$-source form $\omega+\mathrm{E}(\eta)$ which is not globally variational.

São Paulo J.Math.Sci. 5, 1 (2011), 23 35 


\section{References}

[1] I. M. Anderson, The Variational Bicomplex, To Appear.

[2] V. I. Arnold, V. V. Kozlov, And A. I. Neishtadt, Dynamical Systems III, vol. 3 of Encyclopaedia of Mathematical Sciences, Springer-Verlag, New York, 1988, ch. Mathematical Aspects of Classical and Celestial Mechanics, pp. 1-286.

[3] J. Douglas, Solution of the inverse problem of the calculus of variations, Proc. Nat. Acad. Sci. U.S.A., 25 (1939), pp. 631-637.

[4] Solution of the inverse problem of the calculus of variations, Trans. Amer. Math. Soc., 50 (1941), pp. 71-128.

[5] D. A. Gomes, Hamilton-Jacobi methods for vakonomic mechanics, NoDEA: Nonlinear Differential Equations And Applications, 14 (2007), pp. 233-257.

[6] H. Helmholtz, Uber der physikalische bedeutung des princips der kleinsten wirkung, J. Reine Angew. Math., 100 (1887), pp. 137-166.

[7] V. V. KozLov, Dynamics of systems with non-integrable constraints. I, Moscow University Mechanics Bulletin, 37 (1982), pp. 27-34.

[8] - Dynamics of systems with non-integrable constraints. II, Moscow University Mechanics Bulletin, 37 (1982), pp. 74-80.

[9] Dynamics of systems with non-integrable constraints. III, Moscow University Mechanics Bulletin, 38 (1983), pp. 40-51.

[10] I. KuPKA AND W. M. OLIVA, The non-holonomic mechanics, Journal of Differential Equations, 169 (2001), pp. 169-189.

[11] W. M. Oliva, Geometric Mechanics, vol. 1798 of Lecture Notes in Mathematics, Springer-Verlag, 2002.

[12] D. J. Saunders, The Geometry of Jet Bundles, Cambridge University Press, 1989.

[13] F. TAKEns, A global version of the inverse problem of the calculus of variations, Journal of Differential Geometry, 14 (1979), pp. 543-562.

[14] G. TERrA AND M. H. KobAYASHI, On classical mechanical systems with nonlinear constraints, Journal of Geometry and Physics, 49 (2004), pp. 385-417.

[15] - On the variational mechanics with non-linear constraints, Journal de Mathématiques Pures et Appliquées, 83 (2004), pp. 629-671.

[16] T. TsujIshita, On variation bicomplexes associated to differential equations, Osaka J. Math., 19 (1982), pp. 311-363.

[17] W. M. TulczyJEW, The Euler-Lagrange resolution, vol. 836 of Lecture Notes in Mathematics, Springer-Verlag, New York, 1980, pp. 22-48.

[18] M. M. VAinberg, Variational Methods for the Study of Nonlinear Operators, Holden-Day, San Francisco, 1964.

[19] A. M. VinogRAdov, On the algebra-geometric foundation of Lagrangian field theory, Sov. Math. Dokl., 18 (1977), pp. 1200-1204.

[20] - The c-spectral sequence, Lagrangian formalism and conservation laws $i$, ii, J. Math. Anal. Appl., 100 (1984), pp. 1-129.

[21] V. Volterra, Leçons sur les Fonctions de Lignes, Gautherier-Villar, Paris, 1913. 